\title{
Relative asymptotic equivalence of dynamic equations on time scales
}

\author{
Cosme Duque 1 , Hugo Leiva ${ }^{2}$ and Abdessamad Tridane ${ }^{3^{*}}$ (1)
}

"Correspondence:

a-tridane@uaeu.ac.ae

${ }^{3}$ Department Mathematical

Sciences, United Arab Emirates

University, Al Ain, UAE

Full list of author information is

available at the end of the article

\section{Springer}

\begin{abstract}
This paper aims to study the relative equivalence of the solutions of the following dynamic equations $y^{\Delta}(t)=A(t) y(t)$ and $x^{\Delta}(t)=A(t) x(t)+f(t, x(t))$ in the sense that if $y(t)$ is a given solution of the unperturbed system, we provide sufficient conditions to prove that there exists a family of solutions $x(t)$ for the perturbed system such that $\|y(t)-x(t)\|=o(\|y(t)\|)$, as $t \rightarrow \infty$, and conversely, given a solution $x(t)$ of the perturbed system, we give sufficient conditions for the existence of a family of solutions $y(t)$ for the unperturbed system, and such that $\|y(t)-x(t)\|=o(\|x(t)\|)$, as $t \rightarrow \infty$; and in doing so, we have to extend Rodrigues inequality, the Lyapunov exponents, and the polynomial exponential trichotomy on time scales.
\end{abstract}

MSC: Primary 34N05; secondary 34D09

Keywords: Relative asymptotic equivalence; Dynamic equations on time scales; Rodrigues inequality; Lyapunov exponent; Polynomial exponential trichotomy; Contraction mapping theorem

\section{Introduction}

In the study of nature, in particular, in physics, engineering, and economics, among other fields, there are phenomena that vary continuously or discretely, where each phenomenon can be modeled by a differential or difference equation. However, there exists also the possibility that these phenomena can vary both continuously and discretely. The theory of time scales calculus made it possible to create models to study such mixed phenomena, and it also turns out to be a powerful tool in continuous and discrete analysis from a unified point of view (see, for instance, $[2,4,5,7,8]$ and the reference therein). A time scale, which is denoted by $\mathbb{T}$, is any closed nonempty subset of the real numbers, for instance, $\mathbb{N}, \mathbb{Z}$, and $q^{\mathbb{Z}}$ for $q>0$ are times scales, and was introduced by S. Hilger [12, 14] in order to create the theory of dynamic equations that allows unifying differential and difference equations, as well as their extensions, from the same perspective (see $[4,5])$. For example, if $f^{\Delta}$ represents the derivative for a function $f$ defined on $\mathbb{T}$, then it turns out that $f^{\Delta}=f^{\prime}$, the usual derivative, if $\mathbb{T}=\mathbb{R}$, and $f^{\Delta}=\Delta f$, the usual forward difference operator, if $\mathbb{T}=\mathbb{Z}$. In the last years the qualitative study of the solutions of dynamic equations on time scales has been attracting the interest and effort of many mathematicians; in particular, the study of stability, existence of dichotomies, existence of bounded solutions, the existence of peri-

(c) The Author(s) 2022. This article is licensed under a Creative Commons Attribution 4.0 International License, which permits use sharing, adaptation, distribution and reproduction in any medium or format, as long as you give appropriate credit to the original author(s) and the source, provide a link to the Creative Commons licence, and indicate if changes were made. The images or other third party material in this article are included in the article's Creative Commons licence, unless indicated otherwise in a credit line to the material. If material is not included in the article's Creative Commons licence and your intended use is not permitted by statutory regulation or exceeds the permitted use, you will need to obtain permission directly from the copyright holder. To view a copy of this licence, visit http://creativecommons.org/licenses/by/4.0/. 
odic and almost periodic solutions, have been treated by several researchers (for instance, see $[3,9,11,17,18,20-22,25-27]$ and the references therein), however, to the best to our knowledge, the study of asymptotic equivalence of solutions of dynamic equations on time scales has not been carried out. In the case of ordinary differential equations, functional differential equations, and difference equations, we can find works in this direction, see, for instance, $[6,15,16,23,24]$ and the reference therein.

Motivated by this fact, in this paper we shall investigate the relative asymptotic equivalence of the solutions of the following two dynamic equations on time scales:

$$
\begin{aligned}
& y^{\Delta}(t)=A(t) y(t), \quad t \in\left[t_{0}, \infty\right)_{\mathbb{T}}, y \in \mathbb{X}, \\
& x^{\Delta}(t)=A(t) x(t)+f(t, x(t)), \quad t \in\left[t_{0}, \infty\right)_{\mathbb{T}}, x \in \mathbb{X},
\end{aligned}
$$

where $(\mathbb{X},\|\cdot\|)$ is a Banach space, the operator $A: \mathbb{T} \longrightarrow L(\mathbb{X})$ is rd-continuous, $f: \mathbb{T} \times$ $\mathbb{X} \rightarrow \mathbb{X}$ is an rd-continuous function which is a small perturbation in some sense with $f(t, 0)=0$ and

$$
\|f(t, z)-f(t, w)\| \leq h(t)\|z-w\|, \quad t \in\left[t_{0}, \infty\right)_{\mathbb{T}}, z, w \in \mathbb{X},
$$

for $h:\left[t_{0}, \infty\right)_{\mathbb{T}} \longrightarrow \mathbb{R}^{+}$an rd-continuous function.

We will understand this equivalence in the following sense: given a solution $y(t)$ of system (1), there exists a family of solutions $x(t)$ of system (2) such that $\|y(t)-x(t)\|=$ $o(\|y(t)\|)$, as $t \rightarrow \infty$, and conversely, if $x(t)$ is a solution of (2), then there exists a family of solutions $y(t)$ for the unperturbed system (1) such that $\|y(t)-x(t)\|=o(\|x(t)\|)$, as $t \rightarrow \infty$.

It is important to mention that, if $\mathbb{T}=\mathbb{R}$, we get the equations studied in [16], while if $\mathbb{T}=$ $\mathbb{N} \cup\{0\}$ then we have the equations treated in [15]. In both papers, the main tools used were a concept of polynomial exponential dichotomy and the so-called Rodrigues inequality (see also [24] and [19]), which is a generalization of Gronwall's inequality. Concretely, we will treat the following problems:

The direct problem Let $y(t)$ be a solution of (1), with $y(t) \neq 0$, for sufficiently large $t$. Then, does there exist a solution $x(t)$ of (2) such that the relative error satisfies

$$
\lim _{t \rightarrow \infty} \frac{\|y(t)-x(t)\|}{\|y(t)\|}=0 ?
$$

Converse problem Let $x(t)$ be a solution of (2), with $x(t) \neq 0$, for sufficiently large $t$. Then, does there exist a solution $y(t)$ of (1) such that the relative error satisfies

$$
\lim _{t \rightarrow \infty} \frac{\|y(t)-x(t)\|}{\|x(t)\|}=0 ?
$$

To solve these problems, we will extend the Rodrigues inequality, the definition of Lyapunov exponents, and we introduce the definition of polynomial exponential trichotomy on time scales.

The paper is organized as follows. In the next section, we present some fundamentals results about time scales. In Sect. 3 we analyze the direct problem, and in Sect. 4 we study the converse problem. Section 5 is devoted to an example to illustrate our results. We end this work with a remark. 


\section{Preliminaries}

A time scale $\mathbb{T}$ is an arbitrary nonempty closed subset of $\mathbb{R}$. We will assume that $\mathbb{T}$ has the topology inherited from the standard topology of the real numbers. The time scale interval $[a, b]_{\mathbb{T}}$ is defined as $[a, b]_{\mathbb{T}}=\{t \in \mathbb{T}: a \leq t \leq b\}$, with $a, b \in \mathbb{T}$, and open intervals and neighborhoods are defined similarly. The forward jump operator $\sigma: \mathbb{T} \longrightarrow \mathbb{T}$ is defined by $\sigma(t):=\inf \{s \in \mathbb{T}: s>t\}$ and the backward jump operator $\rho: \mathbb{T} \longrightarrow \mathbb{T}$ is defined by $\rho(t):=\sup \{s \in \mathbb{T}: s<t\}$. We put $\inf \emptyset=\sup \mathbb{T}$ (i.e., $\sigma(t)=t$ if $\mathbb{T}$ has a maximum $t$ ) and $\sup \emptyset=\inf \mathbb{T}$ (i.e., $\rho(t)=t$ if $\mathbb{T}$ has a minimum $t$ ), where $\emptyset$ denotes the empty set. A point $t \in \mathbb{T}$ is said to be right-dense if $\sigma(t)=t$, right-scattered if $\sigma(s)>t$, left-dense if $\rho(t)=t$, left-scattered if $\rho(t)<t$, isolated if $\rho(t)<t<\sigma(t)$. The function $\mu: t \longrightarrow[0, \infty)$ defined by $\mu(t):=\sigma(t)-t$ is known as graininess function.

We define the set $\mathbb{T}^{\kappa}$ by

$$
\mathbb{T}^{\kappa}:= \begin{cases}\mathbb{T} \backslash(\rho(\sup \mathbb{T}), \sup \mathbb{T}], & \text { if } \sup \mathbb{T}<\infty \\ \mathbb{T}, & \text { if } \sup \mathbb{T}=\infty\end{cases}
$$

We shall say that a function $f: \mathbb{T} \longrightarrow \mathbb{X}$ is right dense continuous, or just rd-continuous, if

(a) $f$ is continuous at every right-dense point $t \in \mathbb{T}$,

(b) $\lim _{s \rightarrow t^{-}} f(s)$ exists (finite) for every left-dense point $t \in \mathbb{T}$.

The set of rd-continuous functions $f: \mathbb{T} \longrightarrow \mathbb{X}$ will be denoted by $C_{r d}(\mathbb{T}, \mathbb{X})$. A function $f: \mathbb{T} \times \mathbb{X} \longrightarrow \mathbb{X}$ is called rd-continuous if $g$ defined by $g(t)=f(t, x(t))$ is rd-continuous for any continuous function $x: \mathbb{T} \rightarrow \mathbb{X}$.

A function $f: \mathbb{T} \longrightarrow \mathbb{X}$ is called delta differentiable (or simply differentiable) at $t \in \mathbb{T}^{\kappa}$ provided there exists a number $f^{\Delta}(t)$ with the property that given any $\varepsilon>0$, there is a neighborhood $U=(t-\delta, t+\delta)_{\mathbb{T}}$ for some $\delta>0$ such that $\left\|f(\sigma(t))-f(s)-f^{\Delta}(t)(\sigma(t)-s)\right\| \leq$ $\varepsilon|\sigma(t)-s|$ for all $s \in U$, in this case the number $f^{\Delta}(t)$ will be call the delta derivative of $f$ at $t$.

If there exists a function $F: \mathbb{T} \longrightarrow \mathbb{X}$ such that $F^{\Delta}(t)=f(t), t \in \mathbb{T}^{\kappa}$, then $F$ is called an antiderivative of $f$ and the Cauchy integral is defined by

$$
\int_{s}^{t} f(\tau) \Delta \tau=F(t)-F(s), \quad t, s \in \mathbb{T} .
$$

From Theorem 1.74 in [4], we have that every rd-continuous function has an antiderivative, and if $F(t)=\int_{s}^{t} f(\tau) \Delta \tau$, then $F^{\Delta}(t)=f(t), t \in \mathbb{T}^{\kappa}$, i.e., $F$ is an antiderivative of $f$.

A function $p: \mathbb{T} \rightarrow \mathbb{R}$ is said to be regressive (resp. positively regressive) if $1+\mu(t) p(t) \neq$ 0 (resp. $1+\mu(t) p(t)>0), t \in \mathbb{T}$ and the equation

$$
x^{\Delta}=p(t) x, \quad x\left(t_{0}\right)=1
$$

has a unique solution $x(t)=e_{p}\left(t, t_{0}\right)=\exp \int_{t_{0}}^{t} \xi_{\mu(\tau)}(p(\tau)) \Delta \tau$, where

$$
\xi_{\mu}(z):= \begin{cases}\frac{1}{\mu} \log (1+\mu z), & \text { if } \mu>0, \\ z, & \text { if } \mu=0,\end{cases}
$$

The function $e_{p}(t, s)$ satisfies the following properties (see [4, Theorem 2.36]): 
(1) $e_{p}(t, s) e_{q}(t, s)=e_{p \oplus q}(t, s)$,

(2) $\frac{e_{p}(t, s)}{e_{q}(t, s)}=e_{p \ominus q}(t, s)$

(3) $e_{p}(\sigma(t), s)=(1+\mu(t) p(t)) e_{p}(t, s)$,

(4) $e_{p}(t, r) e_{p}(r, s)=e_{p}(t, s)$,

where $(p \oplus q)(t):=p(t)+q(t)+\mu(t) p(t) q(t)$ and $(p \ominus q)(t):=\frac{p(t)-q(t)}{1+\mu(t) q(t)}$.

We will denote by $\mathcal{R}$ (resp. $\mathcal{R}^{+}$) the set of all regressive and rd-continuous functions (resp. positively regressive and rd-continuous).

A mapping $A: \mathbb{T} \longrightarrow L(\mathbb{X})$ is said to be regressive if $I+\mu(t) A(t)$ is invertible for every $t \in \mathbb{T}$.

Henceforth we will suppose that $\sup \{\mathbb{T}\}=\infty$. Since $A$ is rd-continuous, we have that $A$ generates an evolution operator family $T(t, s)=e_{A}(t, s)$ with $t \geq s$, and the only solution of (1) with $y(s)=y_{s}$ is given by $y(t)=T(t, s) y_{s}$ (see [10] and [25]). On the other hand, since $f$ is rd-continuous and satisfies condition (3), we have, by Theorem 5.7 in [13], that equation (2), with $x(s)=x_{s}$, has a unique solution defined on $\mathbb{T}$, which is given by

$$
x(t)=T(t, s) x_{s}+\int_{s}^{t} T(t, \sigma(\tau)) f(\tau, x(\tau)) \Delta \tau
$$

\section{The direct problem}

In this section, we just treat the direct problem through Theorem 3.1, but before we will present the definition of polynomial exponential trichotomy on time scales. It is important to mention that, in the case of dichotomies on time scales, the initial contributions are by Pötzsche in [20,21].

Definition 3.1 We shall say that equation (1) has a polynomial exponential trichotomy on time scales with respect to $\alpha \in C_{r d}\left(\mathbb{T}, \mathbb{R}^{+}\right)$, bounded by $\inf _{\mathbb{T}}\{\alpha(t)\}>0$, if there exist an integer $N \geq 1, M>0, \varepsilon \in C_{r d}\left(\mathbb{T}, \mathbb{R}^{+}\right)$bounded by $\inf _{\mathbb{T}}\{\varepsilon(t)\}>0$, and complementary projections $S(t), P(t), Q(t), U(t): \mathbb{X} \longrightarrow \mathbb{X}, t \in\left[t_{0}, \infty\right)_{\mathbb{T}}$, such that:

(1) For $t \geq s, P(t) T(t, s)=T(t, s) P(s), Q(t) T(t, s)=T(t, s) Q(s)$, and $S(t) T(t, s)=T(t, s) S(s)$

(2) $\left.\left[I_{\mathbb{X}}+\mu(t) A(t)\right]\right|_{\operatorname{Rang}(U(t))}: \operatorname{Rang}(U(t)) \longrightarrow \operatorname{Rang}(U(\sigma(t)))$ is bijective for all right-scattered $t$

(3) $\left.\left[I_{\mathbb{X}}+\mu(t) A(t)\right]\right|_{\operatorname{Rang}(Q(t))}: \operatorname{Rang}(Q(t)) \longrightarrow \operatorname{Rang}(Q(\sigma(t)))$ is bijective for all right-scattered $t$

(4) For each $l, 0 \leq l \leq N-1$, the following conditions hold:

$$
\begin{aligned}
& \|T(t, s) S(s)\| \leq M e_{\alpha \ominus \varepsilon}(t, s), \quad t \geq s, \\
& \|T(t, s) U(s)\| \leq M e_{\alpha \oplus \varepsilon}(t, s), \quad s \geq t, \\
& \|T(t, s)(P(s)+Q(s))\| \leq M t^{N-1} e_{\alpha}(t, s), \quad t \geq s \geq \beta, \\
& \|T(t, s) P(s)\| \leq M t^{l-1} s^{N-1} e_{\alpha}(t, s), \quad t \geq s \geq \beta, \\
& \|T(t, s) Q(s)\| \leq M t^{l} s^{N-l-1} e_{\alpha}(t, s), \quad s \geq t \geq \beta,
\end{aligned}
$$

where $\beta>0$. If $l=0$ then $P(s)=0$. In this case the projections onto the stable, unstable, and center spaces are contained in $L(\mathbb{X})$. 
Remark 1 Conditions (2) and (3) allow for the evolution operator $T(t, s)$ to be invertible between the ranges of $U$ and $Q$, respectively, for details see Proposition 2.3 in [20].

Definition 3.2 If $\alpha>0$ and $l$ is a nonnegative integer, we shall say that a function $y$ : $\left[t_{0}, \infty\right)_{\mathbb{T}} \longrightarrow \mathbb{X}$ is of order $t^{l} e_{\alpha}\left(t, t_{0}\right)$, and denote it by $y(t) \cong t^{l} e_{\alpha}\left(t, t_{0}\right)$, if

$$
0<\liminf _{t \rightarrow \infty} \frac{\|y(t)\|}{t^{l} e_{\alpha}\left(t, t_{0}\right)} \leq \limsup _{t \rightarrow \infty} \frac{\|y(t)\|}{t^{l} e_{\alpha}\left(t, t_{0}\right)}<\infty .
$$

The following theorem is our main result in this section

Theorem 3.1 Let $y(t)$ be a solution of (1) with $y(t) \cong t^{l} e_{\alpha}\left(t, t_{0}\right)$. Suppose that (3) is satisfied and that equation (1) has a polynomial exponential trichotomy on time scales with respect to $\alpha$ and the function $h(t)$ satisfies

$$
\int_{t_{0}}^{\infty} \sigma(s)^{N-1} h(s) \Delta s<\infty
$$

Then there exists a solution $x(t)$ of (2) such that

$$
\lim _{t \rightarrow \infty} \frac{\|x(t)-y(t)\|}{\|y(t)\|}=0 .
$$

Proof Let us consider the change of variable

$$
z(t):=e_{\ominus \alpha}\left(t, t_{0}\right)(x(t)-y(t))
$$

then we obtain the following equation for $z$ :

$$
z^{\Delta}(t)=A_{\alpha}(t) z(t)+F(t, z(t))
$$

where $A_{\alpha}(t)=\frac{1}{1+\mu(t) \alpha}[-\alpha I+A(t)]$ and $F(t, z)=\frac{e_{\ominus \alpha}\left(t, t_{0}\right)}{1+\mu(t) \alpha} f\left(t, y(t)+e_{\alpha}\left(t, t_{0}\right) z(t)\right)$.

A straightforward computation shows that the evolution operator $T_{\alpha}(t, s)$ generated by $A_{\alpha}(t)$ is given by

$$
T_{\alpha}(t, s)=e_{\ominus \alpha}(t, s) T(t, s), \quad t \geq s
$$

Hence, we obtain the following conditions for $T_{\alpha}(t, s)$ :

$$
\begin{aligned}
& \left\|T_{\alpha}(t, s) P(s)\right\|=\left\|e_{\ominus \alpha}(t, s) T(t, s) P(s)\right\| \leq M t^{l-1} s^{N-1}, \quad t \geq s \geq \beta>0, \\
& \left\|T_{\alpha}(t, s) Q(s)\right\|=\left\|e_{\ominus \alpha}(t, s) T(t, s) Q(s)\right\| \leq M t^{l} s^{N-l-1}, \quad s \geq t \geq \beta .
\end{aligned}
$$

Now,

$$
\lim _{t \rightarrow \infty} \frac{\|x(t)-y(t)\|}{\|y(t)\|}=\lim _{t \rightarrow \infty} \frac{\|x(t)-y(t)\|}{t^{l} e_{\alpha}\left(t, t_{0}\right)}=\lim _{t \rightarrow \infty} \frac{\left\|e_{\ominus \alpha}\left(t, t_{0}\right)(x(t)-y(t))\right\|}{t^{l}}=\lim _{t \rightarrow \infty} \frac{\|z(t)\|}{t^{l}} .
$$

Therefore, the problem is reduced to proving the existence of a solution of (14) such that

$$
\lim _{t \rightarrow \infty} \frac{\|z(t)\|}{t^{l}}=0 .
$$


If we suppose for a moment that $z(t)$ is a solution of (14) such that (16) holds. Then using the variation of constant formula for equation (14) (Theorem 5.8 in [4]), we get that

$$
\begin{aligned}
z(t)= & T_{\alpha}(t, \eta) z(\eta)+\int_{\eta}^{t} T_{\alpha}(t, \sigma(s)) F(s, z(s)) \Delta s \\
= & T_{\alpha}(t, \eta)[P(\eta)+Q(\eta)] z(\eta)+\int_{\eta}^{t} T_{\alpha}(t, \sigma(s))[P(\sigma(s))+Q(\sigma(s))] F(s, z(s)) \Delta s \\
= & T_{\alpha}(t, \eta) P(\eta) z(\eta)+T_{\alpha}(t, \eta) Q(\eta) z(\eta)+\int_{\eta}^{t} T_{\alpha}(t, \sigma(s)) P(\sigma(s)) F(s, z(s)) \Delta s \\
& +\int_{\eta}^{\tau} T_{\alpha}(t, \sigma(s)) Q(\sigma(s)) F(s, z(s)) \Delta s-\int_{t}^{\tau} T_{\alpha}(t, \sigma(s)) Q(\sigma(s)) F(s, z(s)) \Delta s .
\end{aligned}
$$

If we let $\tau \longrightarrow \infty$, then we get

$$
\begin{aligned}
z(t)= & T_{\alpha}(t, \beta) P(\beta) z(\beta)+T_{\alpha}(t, \beta) Q(\beta) z(\beta)+\int_{\beta}^{t} T_{\alpha}(t, \sigma(s)) P(\sigma(s)) F(s, z(s)) \Delta s \\
= & T_{\alpha}(t, \eta)\left[Q(\eta) z(\eta)+\int_{\eta}^{\infty} T_{\alpha}(\eta, \sigma(s)) Q(\sigma(s)) F(s, z(s)) \Delta s\right]+T(t, \eta) P(\eta) z(\eta) \\
& +\int_{\eta}^{t} T_{\alpha}(\eta, \sigma(s)) P(\sigma(s)) F(s, z(s)) \Delta s-\int_{t}^{\infty} T_{\alpha}(t, \sigma(s)) Q(\sigma(s)) F(s, z(s)) \Delta s .
\end{aligned}
$$

Note that

$$
\int_{\eta}^{\infty} T_{\alpha}(\eta, s(s)) Q(\sigma(s)) F(s, z(s)) \Delta s<\infty .
$$

In fact, since

$$
\begin{aligned}
\|F(t, z)\| & =\left\|\frac{e_{\ominus \alpha}\left(t, t_{0}\right)}{1+\mu(t) \alpha} f\left(t, y(t)+e_{\alpha}\left(t, t_{0}\right) z(t)\right)\right\| \\
& \leq e_{\ominus \alpha}\left(t, t_{0}\right) h(t)\left\|y(t)+e_{\alpha}\left(t, t_{0}\right) z(t)\right\| \leq e_{\ominus \alpha}\left(t, t_{0}\right) h(t)\|y(t)\|+h(t)\|z(s)\|,
\end{aligned}
$$

we obtain

$$
\begin{aligned}
& \left\|\int_{\eta}^{\infty} T_{\alpha}(\eta, \sigma(s)) Q(\sigma(s)) F(s, z(s)) \Delta s\right\| \\
& \quad \leq \int_{\eta}^{\infty}\left\|T_{\alpha}(\eta, \sigma(s)) Q(\sigma(s))\right\|\|F(s, z(s))\| \Delta s \\
& \quad \leq \int_{\eta}^{\infty} M \eta^{l} \sigma^{N-l-1}(s)\left[e_{\ominus \alpha}(s) h(s)\|y(s)\|+h(s)\|z(s)\|\right] \Delta s \\
& \quad \leq M \eta^{l}\left[\int_{\eta}^{\infty} \sigma^{N-l-1}(s) h(s) s^{l} \Delta s+\int_{\eta}^{\infty} \sigma^{N-1}(s) h(s) \frac{\|z(s)\|}{\sigma^{l}(s)} \Delta s\right] \\
& \quad \leq M \eta^{l}\left[\int_{\eta}^{\infty} \sigma^{N-1}(s) h(s) \Delta s+\int_{\eta}^{\infty} \sigma^{N-1}(s) h(s) \frac{\|z(s)\|}{s^{l}} \Delta s\right]<\infty
\end{aligned}
$$


As it will become clear in the next calculation, condition (16) is satisfied if we require

$$
Q(\eta) z(\eta)+\int_{\eta}^{\infty} T_{\alpha}(\eta, \sigma(s)) Q(\sigma(s)) F(s, z(s)) \Delta s=0
$$

So, we get that

$$
\begin{aligned}
z(t)= & T_{\alpha}(t, \eta) P(\eta) z(\eta)+\int_{\eta}^{\infty} T_{\alpha}(t, \sigma(s)) P(\sigma(s)) F(s, z(s)) \Delta s \\
& -\int_{t}^{\infty} T_{\alpha}(t, \sigma(s)) Q(\sigma(s)) F(s, z(s)) \Delta s,
\end{aligned}
$$

for $t \geq \eta$.

Conversely, if $z(t)$, with $t^{-l} z(t)$ bounded, satisfies the integral equation (17), then $z$ satisfies equation (14). Now, to complete the proof, we shall prove that the integral equation (17) has a solution in the Banach space

$$
Z_{l}=\left\{z \in C_{r d}\left([\eta, \infty)_{\mathbb{T}}, \mathbb{X}\right):\|z\|_{l}=\sup _{t>\eta} t^{-1}\|z(t)\|<\infty\right\} .
$$

We define the operator $\Gamma: Z_{l} \longrightarrow Z_{l}$ by

$$
\begin{aligned}
(\Gamma z)(t):= & T_{\alpha}(t, \eta) P(\eta) \omega+\int_{\eta}^{t} T_{\alpha}(t, \sigma(s)) P(\sigma(s)) F(s, z(s)) \Delta s \\
& -\int_{t}^{\infty} T_{\alpha}(t, \sigma(s)) Q(\sigma(s)) F(s, z(s)) \Delta s, \quad t \geq \eta .
\end{aligned}
$$

Next, we shall prove that $\Gamma$ maps $Z_{l}$ into $Z_{l}$. In fact, for $z \in Z_{l}$ we have the following estimate:

$$
\begin{aligned}
\|(\Gamma z)(t)\| \leq & \left\|T_{\alpha}(t, \eta) P(\eta)\right\|\|\omega\|+\int_{\eta}^{t}\left\|T_{\alpha}(t, \sigma(s)) P(\sigma(s))\right\| \| F(s, z(s) \| \Delta s \\
& +\int_{t}^{\infty}\left\|T_{\alpha}(t, \sigma(s)) Q(\sigma(s))\right\| \| F(s, z(s) \| \Delta s \\
\leq & M t^{l-1} \eta^{N-l}\|\omega\|+\int_{\eta}^{t} M t^{l-1} \sigma^{N-l}(s)\left[e_{\ominus \alpha}\left(s, t_{0}\right) h(s)\|y(s)\|+h(s)\|z(s)\|\right] \Delta s \\
& +\int_{t}^{\infty} M t^{l} \sigma^{N-l-1}(s)\left[e_{\ominus \alpha}\left(s, t_{0}\right) h(s)\|y(s)\|+h(s)\|z(s)\|\right] \Delta s,
\end{aligned}
$$

and then

$$
\begin{aligned}
t^{-l}\|(\Gamma z)(t)\| \leq & \frac{M \eta^{N-l}}{t}\|\omega\|+\frac{M}{t} \int_{\eta}^{t} \frac{\sigma^{N}(s)}{\sigma^{l}(s)} e_{\ominus \alpha}\left(s, t_{0}\right) h(s)\|y(s)\| \Delta s \\
& +\frac{M}{t} \int_{\eta}^{t} \frac{\sigma^{N}(s)}{\sigma^{l}(s)} h(s)\|z(s)\| \Delta s+M \int_{t}^{\infty} \frac{\sigma^{N-1}(s)}{\sigma^{l}(s)} e_{\ominus \alpha}\left(s, t_{0}\right) h(s)\|y(s)\| \Delta s \\
& +M \int_{t}^{\infty} \frac{\sigma^{N-1}(s)}{\sigma^{l}(s)} h(s)\|z(s)\| \Delta s
\end{aligned}
$$




$$
\begin{aligned}
\leq & \frac{M \eta^{N-l}}{t}\|\omega\|+\frac{M\|y\|_{\alpha, l}}{t} \int_{\eta}^{t} \sigma^{N}(s) h(s) \Delta s+\frac{M\|z\|_{l}}{t} \int_{\eta}^{t} \sigma^{N}(s) h(s) \Delta s \\
& +M\|y\|_{\alpha, l} \int_{t}^{\infty} \sigma^{N-1}(s) h(s) \Delta s+M\|z\|_{l} \int_{t}^{\infty} \sigma^{N-1}(s) h(s) \Delta s,
\end{aligned}
$$

where $\|y\|_{\alpha, l}:=\sup _{t \geq \eta} t^{-l} e_{\ominus \alpha}\left(t, t_{0}\right)\|y(t)\|$. Therefore, $\lim _{t \rightarrow \infty} \frac{\|(\Gamma z)(t)\|}{t^{l}}=0$, and so $\Gamma z \in Z_{l}$. Now, we shall prove that the operator $\Gamma$ is a contraction mapping. In fact, let $z_{1}, z_{2} \in Z_{l}$ and consider

$$
\begin{aligned}
t^{-1}\left\|\left(\Gamma z_{1}\right)(t)-\left(\Gamma z_{2}\right)(t)\right\| \leq & t^{-l}\left[\int_{\eta}^{t}\left\|T_{\alpha}(t, \sigma(s)) P(\sigma(s))\right\|\left\|F\left(s, z_{1}(s)\right)-F\left(s, z_{2}(s)\right)\right\| \Delta s\right. \\
& \left.+\int_{t}^{\infty}\left\|T_{\alpha}(t, \sigma(s)) Q(\sigma(s))\right\|\left\|F\left(s, z_{1}(s)\right)-F\left(s, z_{2}(s)\right)\right\| \Delta s\right] \\
\leq & \frac{M}{t} \int_{\eta}^{t} \sigma^{N}(s) h(s) \sigma^{-l}(s)\left\|z_{1}(s)-z_{2}(s)\right\| \Delta s \\
& +M \int_{\eta}^{\infty} \sigma^{N-1}(s) h(s) \sigma^{-l}(s)\left\|z_{1}(s)-z_{2}(s)\right\| \Delta s \\
\leq & \frac{M}{t} \int_{\eta}^{t} \sigma^{N}(s) h(s)\left\|z_{1}-z_{2}\right\|_{l} \Delta s \\
& +M \int_{t}^{\infty} \sigma^{N-1} h(s)\left\|z_{1}-z_{2}\right\|_{l} \Delta s \\
\leq & M\left[\int_{\eta}^{\infty} \sigma^{N-1}(s) h(s) \Delta s\right]\left\|z_{1}-z_{2}\right\|_{l} .
\end{aligned}
$$

We choose $\eta$ large enough such that $\int_{\eta}^{\infty} \sigma^{N-1}(s) h(s) \Delta s<1 / M$.

Therefore, we get that $\Gamma$ is a contraction mapping from $Z_{l}$ to $Z_{l}$. By applying the contraction mapping principle, it follows that this operator has a unique fixed point which depends on $\omega \in \mathbb{X}$. The solution $x(t)$ of (2) that solves the Direct Problem, is given by $x(t):=e_{\alpha}\left(t, t_{0}\right) z(t)+y(t)$, where $z(\cdot)$ is the fixed point of $\Gamma$. The above estimates also imply that $t^{-l}\|z(t)\| \longrightarrow 0$, as $t \longrightarrow \infty$.

\section{The converse problem}

In the present section we will study the converse problem, as a special case. First, we will present the Rodrigues inequality on time scales. We need the following lemma.

Lemma 4.1 Let $\beta$ and $\varphi$ be nonnegative and $r d$-continuous functions defined on $\left[t_{0}, \infty\right)_{\mathbb{T}}$ with $-\varphi \in \mathcal{R}^{+}$and such that

$$
\int_{t_{0}}^{\infty} \ominus(-\varphi(s)) \Delta s=\int_{t_{0}}^{\infty} \frac{\varphi(s)}{1-\mu(s) \varphi(s)} \Delta s<\infty
$$

If $u(t) \geq 0$ is a bounded $r d$-continuous and decreasing function defined for $t \in\left[t_{0}, \infty\right)_{\mathbb{T}}$ and satisfies

$$
u(t) \leq \beta(t)+\int_{t}^{\infty} \varphi(s) u(s) \Delta s, \quad t>t_{0}
$$


then

$$
u(t) \leq \beta(t) \exp \left\{\int_{t}^{\infty} \ominus(-\varphi(s)) \Delta s\right\}
$$

Proof Given $\varepsilon>0$, there exists $M>0$ such that if $\tau \geq M$ then

$$
\int_{t}^{\infty} \varphi(s) u(s) \Delta s<\int_{t}^{\tau} \varphi(s) u(s) \Delta s+\varepsilon, \quad \text { if } \tau \geq M .
$$

For $\tau>M$, suppose first that $\beta(t)=c$ is constant, then

$$
u(t) \leq(c+\varepsilon)+\int_{t}^{\tau} \varphi(s) u(s) \Delta s
$$

Let us define $z(t)=(c+\varepsilon)+\int_{t}^{\tau} \varphi(s) u(s) \Delta s$, then $z^{\Delta}(t)=-\varphi(t) u(t) \geq-\varphi(t) z(t)$. Now, a straightforward computation shows that

$$
\left[z e_{-\varphi}(\tau, \cdot)\right]^{\Delta}(t)=\left[z^{\Delta}(t)+\varphi(t) z(t)\right] e_{-\varphi}(\tau, \sigma(t)) \geq 0
$$

therefore $z(\tau)-z(t) e_{-\varphi}(\tau, t) \geq 0$, and so $z(\tau) \geq z(t) e_{-\varphi}(\tau, t)$, hence $z(t) \leq z(\tau) e_{\ominus(-\varphi)}(\tau, t)$, which implies that $z(t) \leq(c+\varepsilon) e_{\ominus(-\varphi)}(\tau, t)$, and hence

$$
z(t) \leq(c+\varepsilon) \exp \left\{\int_{t}^{\tau} \xi_{\mu(s)}(\ominus(-\varphi(s))) \Delta s\right\}
$$

Letting $\tau \longrightarrow \infty$ and from arbitrariness of $\varepsilon$, we have that

$$
z(t) \leq c \exp \left\{\int_{t}^{\infty} \xi_{\mu(s)}(\ominus(-\varphi(s)) \Delta s\}=c e_{\ominus(-\varphi)}(\infty, t)\right.
$$

Since $u(t) \leq z(t)$, then

$$
u(t) \leq c e_{\ominus(-\varphi)}(\infty, t) .
$$

Using Lemma 1.1.1 in [1] yields

$$
u(t) \leq c \exp \left\{\int_{t}^{\infty} \ominus(-\varphi(s)) \Delta s\right\}
$$

Now, let $u(t)=\beta(t) w(t)$. Then

$$
\beta(t) w(t) \leq \beta(t)+\int_{t}^{\infty} \varphi(s) \beta(s) w(s) \Delta s
$$

Thus,

$$
w(t) \leq 1+\int_{t}^{\infty} \varphi(s) \frac{\beta(s)}{\beta(t)} w(s) \Delta s \leq 1+\int_{t}^{\infty} \varphi(s) w(s) \Delta s
$$


which, by considering (20), gives

$$
w(t) \leq \exp \left\{\int_{t}^{\infty} \ominus(-\varphi(s)) \Delta s\right\}
$$

So

$$
u(t) \leq \beta(t) \exp \left\{\int_{t}^{\infty} \ominus(-\varphi(s)) \Delta s\right\} .
$$

Lemma 4.2 (Rodrigues inequality) Let $f$ and $g$ be nonnegative $r d$-continuous functions defined for $t \in\left[t_{0}, \infty\right)_{\mathbb{T}}$, with $-g \in \mathcal{R}^{+}$. Let $\gamma(t)>0$ be a decreasing $r d$-continuous function, for $t \geq \eta$ and $\eta$ sufficiently large, in such a way that

$$
\beta=\int_{\eta}^{\infty} g(s) \Delta s+\int_{\eta}^{\infty} f(s) \Delta s<1 .
$$

Suppose that $u$ is a nonnegative continuous function such that $\gamma u$ is bounded and

$$
u(t) \leq c+\int_{\eta}^{t} f(s) u(s) \Delta s+\frac{1}{\gamma(t)} \int_{t}^{\infty} \gamma(s) g(s) u(s) \Delta s
$$

for $t \geq \eta$, where $c \geq 0$ is a constant. Then, for $t \in[0, \infty)_{\mathbb{T}}$,

$$
u(t) \leq \frac{c}{1-\beta} \exp \left\{\frac{1}{1-\beta} \int_{t}^{\infty} \ominus(-g(s)) \Delta s\right\} .
$$

Proof Let

$$
v(t)=\max _{s \in[\eta, t]_{\mathbb{T}}} u(s) .
$$

Then $v(t)$ is an increasing continuous function such that $u(t) \leq v(t)$ and $\gamma(t) v(t)$ is bounded for $t \in\left[t_{0}, \infty\right)_{\mathbb{T}}$. For a given $t \geq \eta$, there exists $t_{1} \in[\eta, t]_{\mathbb{T}}$ satisfying $v(t)=u\left(t_{1}\right)$. This implies

$$
v(t) \leq c+\int_{\eta}^{t_{1}} f(s) v(s) \Delta s+\frac{1}{\gamma\left(t_{1}\right)} \int_{t_{1}}^{\infty} \gamma(s) g(s) v(s) \Delta s
$$

But

$$
\begin{aligned}
\int_{t_{1}}^{\infty} \gamma(s) g(s) v(s) \Delta s & =\int_{t_{1}}^{t} \gamma(s) g(s) v(s) \Delta s+\int_{t}^{\infty} \gamma(s) g(s) v(s) \Delta s \\
& \leq \gamma\left(t_{1}\right) v(t) \int_{\eta}^{\infty} g(s) \Delta s+\int_{t}^{\infty} \gamma(s) g(s) v(s) \Delta s .
\end{aligned}
$$

Combining the above inequalities, we get

$$
v(t) \leq c+v(t)\left[\int_{\eta}^{\infty} f(s) \Delta s+\int_{\eta}^{\infty} g(s) \Delta s\right]+\frac{1}{\gamma(t)} \int_{t}^{\infty} \gamma(s) g(s) v(s) \Delta s .
$$


Then,

$$
\gamma(t) v(t) \leq \frac{1}{1-\beta}\left[c \gamma(t)+\int_{t}^{\infty} \gamma(s) g(s) v(s) \Delta s\right] .
$$

By using Lemma 4.1, it follows that

$$
\gamma(t) v(t) \leq \frac{c}{1-\beta} \gamma(t) \exp \left\{\frac{1}{1-\beta} \int_{t}^{\infty} \ominus(-g(s)) \Delta s\right\} .
$$

So

$$
u(t) \leq \frac{c}{1-\beta} \exp \left\{\frac{1}{1-\beta} \int_{t}^{\infty} \ominus(-g(s)) \Delta s\right\} .
$$

Definition 4.1 Let $y(t)$ be a solution of (1). We say that $\lambda>0$ is a Lyapunov exponent of $y(t)$ if, given $\varepsilon>0$, then there exist $T \in\left[t_{0}, \infty\right)_{\mathbb{T}}$ and $L>0$ such that

$$
L e_{\lambda-\varepsilon}\left(t, t_{0}\right) \leq\|y(t)\| \leq L e_{\lambda+\varepsilon}\left(t, t_{0}\right), \quad t \in[T, \infty)_{\mathbb{T}} .
$$

Remark 2 It is easy to see that (21) implies that

$$
\varlimsup_{t \rightarrow \infty} \frac{\log (\|y(t)\|)}{t-t_{0}}=\varlimsup_{t \rightarrow \infty} \frac{1}{t-t_{0}} \int_{t_{0}}^{t} \lim _{s \searrow \mu(\tau)} \frac{\log (1+s \lambda)}{s} \Delta \tau=\gamma(\lambda) .
$$

This $\gamma(\lambda)$ was used in [22] to characterize stability of linear systems on time scales.

Lemma 4.3 Let $T(t, s)$ be the evolution operator generated by $A(t)$. Assume that there exist complementary projections $P(s), Q(s), \alpha<\beta$, and $K>0$, as in the first part of Definition 3.1, such that

$$
\begin{aligned}
\|T(t, s) P(s)\| \leq K e_{\alpha}(t, s), & t \geq s, \\
\|T(t, s) Q(s)\| \leq K e_{\beta}(t, s), & t \leq s,
\end{aligned}
$$

and

$$
\int_{t_{0}}^{\infty} h(s) \Delta s<\infty
$$

Then

(a) $\|T(t, s) Q(s) x\| \geq K^{-1} e_{\beta}(t, s)\|Q(s) x\|, t \geq s$, for all $x \in \mathbb{X}$,

(b) There is no solution $x(t)$ of (2) with Lyapunov exponent $\lambda$, with $\alpha<\lambda<\beta$.

Proof a) First of all, we note that $T(s, t) Q(t) T(t, s) Q(s) x(t)=Q(s) x(t)$, so we have that

$$
\begin{aligned}
\|Q(s) x(t)\| & =\|T(s, t) Q(t) T(t, s) Q(s) x(t)\| \leq\|T(s, t) Q(t)\|\|T(t, s) Q(s) x(t)\| \\
& \leq K e_{\beta}(s, t)\|T(t, s) Q(s) x(t)\|, \quad t \geq s .
\end{aligned}
$$

Therefore, $\|T(t, s) Q(s) x\| \geq K^{-1} e_{\beta}(t, s)\|Q(s) x\|, t \geq s$. 
b) Let us suppose the existence of a solution $x(t)$ of (2) having Lyapunov exponent $\lambda$ with $\alpha<\lambda<\beta$. Then for $t \geq \eta>0$,

$$
\begin{aligned}
x(t)= & T(t, \eta) x(\eta)+\int_{\eta}^{t} T(t, \sigma(s)) f(s, x(s)) \Delta s \\
= & T(t, \eta) \omega+\int_{\eta}^{t} T(t, \sigma(s)) P(\sigma(s)) f(s, x(s)) \Delta s \\
& -\int_{t}^{\infty} T(t, \sigma(s)) Q(\sigma(s)) f(s, x(s)) \Delta s,
\end{aligned}
$$

where $\omega=x(\eta)+\int_{\eta}^{\infty} T(\eta, \sigma(s)) Q(\sigma(s)) f(s, x(s)) \Delta s$.

If $\delta>0$ is such that $\lambda+\delta<\beta, \lambda-\delta>\alpha$, then we have

$$
\|x(t)\| \leq L e_{\lambda+\delta}\left(t, t_{0}\right)
$$

for $t \geq \eta$ and for a suitable $L \geq 0$. Moreover,

$$
\begin{aligned}
\|T(t, \sigma(s)) Q(\sigma(s)) f(s, x(s))\| & \leq K e_{\beta}(t, \sigma(s)) h(s) L e_{\lambda+\delta}\left(s, t_{0}\right) \\
& =K(1+\mu(s)(\ominus \beta)) e_{\ominus \beta}(s, t) h(s) L e_{\lambda+\delta}\left(s, t_{0}\right) \\
& \leq K L e_{\beta}(t) h(s) e_{\ominus(\beta \ominus(\lambda+\delta))}\left(s, t_{0}\right) .
\end{aligned}
$$

Since $\beta \ominus(\lambda+\delta)=\frac{\beta-(\lambda+\delta)}{1+\mu(s)(\lambda+\delta)}>0$, then $\lim _{s \rightarrow \infty} e_{\ominus(\beta \ominus(\lambda+\delta))}\left(s, t_{0}\right)=0$, therefore the above integrals are convergent. We also have

$$
\begin{aligned}
& e_{\ominus(\lambda+\delta)}\left(t, t_{0}\right) \int_{t}^{\infty}\|T(t, \sigma(s)) Q(\sigma(s)) f(s, x(s))\| \Delta s \\
& \quad \leq e_{\ominus(\lambda+\delta)}\left(t, t_{0}\right) \int_{t}^{\infty}\|T(t, \sigma(s)) Q(\sigma(s))\|\|f(s, x(s)) \Delta s\| \Delta s \\
& \quad \leq e_{\ominus(\lambda+\delta)}\left(t, t_{0}\right) \int_{t}^{\infty} K e_{\beta}(t, \sigma(s)) h(s) L e_{\lambda+\delta}\left(s, t_{0}\right) \Delta s \\
& \quad \leq e_{\beta \ominus(\lambda+\delta)}\left(t, t_{0}\right) \int_{t}^{\infty} K L h(s) e_{\ominus(\beta \ominus(\lambda+\delta))}\left(s, t_{0}\right) \Delta s \\
& \quad \leq K L \int_{t}^{\infty} h(s) \Delta s .
\end{aligned}
$$

Therefore, $e_{\ominus(\lambda+\delta)}\left(t, t_{0}\right) \int_{t}^{\infty} T(t, \sigma(s)) Q(\sigma(s)) f(s, x(s)) \Delta s \longrightarrow 0$, as $t \longrightarrow \infty$. On the other hand,

$$
\begin{gathered}
e_{\ominus(\lambda+\delta)}\left(t, t_{0}\right) \int_{\eta}^{t}\|T(t, \sigma(s)) P(\sigma(s)) f(s, x(s))\| \Delta s \\
\leq e_{\ominus(\lambda+\delta)}\left(t, t_{0}\right) \int_{\eta}^{t}\|T(t, \sigma(s)) P(\sigma(s))\| \| f(s, x(s) \| \Delta s \\
\leq e_{\ominus(\lambda+\delta)}\left(t, t_{0}\right) \int_{\eta}^{t} K e_{\alpha}(t, \sigma(s)) h(s) L e_{\lambda+\delta}\left(s, t_{0}\right) \Delta s
\end{gathered}
$$




$$
\begin{aligned}
& \leq e_{\alpha \ominus(\lambda+\delta)}\left(t, t_{0}\right) \int_{\eta}^{t} K L e_{\ominus(\alpha \ominus(\lambda+\delta))}\left(s, t_{0}\right) h(s) \Delta s \\
& \leq \int_{\eta}^{t} K L h(s) \Delta s .
\end{aligned}
$$

So $e_{\ominus(\lambda+\delta)}\left(t, t_{0}\right) \int_{\eta}^{t} T(t, \sigma(s)) P(\sigma(s)) f(s, x(s)) \Delta s \longrightarrow 0$, as $t \longrightarrow \infty$.

We claim that $Q(\eta) \omega=0$. In fact, let us suppose that $Q(\eta) \omega \neq 0$, then,

$$
\begin{aligned}
e_{\ominus(\lambda+\delta)}\left(t, t_{0}\right)\|T(t, \eta) Q(\eta) \omega\| & \geq e_{\ominus(\lambda+\delta)}\left(t, t_{0}\right) K^{-1} e_{\beta}(t, \eta)\|Q(\eta) \omega\| \\
& \geq e_{\beta \ominus(\lambda+\delta)}\left(t, t_{0}\right) K^{-1} e_{\beta}\left(t_{0}, \eta\right)\|Q(\eta) \omega\| \rightarrow \infty, \quad t \rightarrow \infty .
\end{aligned}
$$

On the other hand

$$
Q(t) x(t)=T(t, \eta) Q(\eta) \omega-\int_{t}^{\infty} T(t, \sigma(s)) Q(\sigma(s)) f(s, x(s)) \Delta s,
$$

Therefore,

$$
\begin{aligned}
& e_{\ominus(\lambda+\delta)}\left(t, t_{0}\right)\|Q(t) x(t)\| \\
& \quad \geq e_{\ominus(\lambda+\delta)}\left(t, t_{0}\right)\|T(t, \eta) Q(\eta) \omega\|-e_{\ominus(\lambda+\delta)}\left(t, t_{0}\right)\left\|\int_{t}^{\infty} T(t, \sigma(s)) Q(\sigma(s)) f(s, x(s)) \Delta s\right\| \\
& \quad \longrightarrow \infty, \quad t \longrightarrow \infty
\end{aligned}
$$

which is a contradiction to (22). Then, we have

$$
\begin{aligned}
x(t)= & T(t, \eta) P(\eta) \omega+\int_{\eta}^{t} T(t, \sigma(s)) P(\sigma(s)) f(s, x(s)) \Delta s \\
& -\int_{t}^{\infty} T(t, \sigma(s)) Q(\sigma(s)) f(s, x(s)) \Delta s .
\end{aligned}
$$

Since

$$
\begin{aligned}
\|T(t, s) P(s)\| \leq K e_{\alpha}(t, s) \leq K e_{\lambda-\delta}(t, s), & t \geq s, \\
\|T(t, s) Q(s)\| \leq K e_{\beta}(t, s) \leq K e_{\lambda+\delta}(t, s), & t \leq s,
\end{aligned}
$$

it follows that

$$
\begin{aligned}
\|x(t)\| \leq & \|T(t, \eta) P(\eta) \omega\|+\int_{\eta}^{t}\|T(t, \sigma(s)) P(\sigma(s)) f(s, x(s))\| \Delta s \\
& +\int_{t}^{\infty}\|T(t, \sigma(s)) Q(\sigma(s)) f(s, x(s))\| \Delta s \\
\leq & K e_{\lambda-\delta}(t, \eta)\|w\|+\int_{\eta}^{t} K e_{\lambda-\delta}(t, \sigma(s)) h(s)\|x(s)\| \Delta s \\
& +\int_{t}^{\infty} K e_{\lambda+\delta}(t, \sigma(s)) h(s)\|x(s)\| \Delta s
\end{aligned}
$$




$$
\begin{aligned}
\leq & K e_{\lambda-\delta}(t, \eta)\|\omega\|+\int_{\eta}^{t} K e_{\lambda-\delta}(t, s) h(s)\|x(s)\| \Delta s \\
& +\int_{t}^{\infty} K e_{\lambda+\delta}(t, s) h(s)\|x(s)\| \Delta s,
\end{aligned}
$$

which implies

$$
\begin{aligned}
& e_{\ominus(\lambda-\delta)}\left(t, t_{0}\right)\|x(t)\| \\
& \leq K e_{\ominus(\lambda-\delta)}\left(\eta, t_{0}\right)\|\omega\|+K \int_{\eta}^{t} h(s) e_{\ominus(\lambda-\delta)}\left(s, t_{0}\right)\|x(s)\| \Delta s \\
& \quad+K e_{(\lambda+\delta) \ominus(\lambda-\delta)}\left(t, t_{0}\right) \int_{t}^{\infty} e_{(\lambda-\delta) \ominus(\lambda+\delta)}\left(s, t_{0}\right) h(s) e_{\ominus(\lambda-\delta)}\left(s, t_{0}\right)\|x(s)\| \Delta s .
\end{aligned}
$$

If we let $u(t):=e_{\ominus(\lambda-\delta)}\left(t, t_{0}\right)\|x(t)\|$, then we obtain

$$
\begin{aligned}
u(t) \leq & K e_{\ominus(\lambda-\delta)}\left(\eta, t_{0}\right)\|\omega\|+K \int_{\eta}^{t} h(s) u(s) \Delta s \\
& +\operatorname{Ke}_{(\lambda+\delta) \ominus(\lambda-\delta)}\left(t, t_{0}\right) \int_{t}^{\infty} e_{(\lambda-\delta) \ominus(\lambda+\delta)}\left(s, t_{0}\right) h(s) u(s) \Delta s .
\end{aligned}
$$

Now, applying the Rodrigues inequality with

$$
g(t)=f(t)=K h(t), \quad \gamma(t)=e_{(\lambda-\delta) \ominus(\lambda+\delta)}\left(t, t_{0}\right),
$$

we get that

$$
u(t) \leq \frac{K e_{\ominus(\lambda-\delta)}\left(\eta, t_{0}\right)\|\omega\|}{1-\beta} \exp \left\{\frac{1}{1-\beta} \int_{t}^{\infty} \ominus(-h(s)) \Delta s\right\}
$$

where $\beta=\int_{\eta}^{\infty} 2 K h(s) \Delta s<1$.

So, $u(t):=e_{\ominus(\lambda-\delta)}\left(t, t_{0}\right)\|x(t)\|$ is bounded for $t \geq \eta$ and this contradicts the hypothesis that the Lyapunov exponent of $x(t)$ is $\lambda$.

Lemma 4.4 Suppose that $T(t, s)$ is the evolution operator generated by $A(t)$. Assume that there exist complementary projections $P(t), Q(t), \alpha<\beta, K>0$, and a positive integer $n$, as in the first part of Definition 3.1, such that

$$
\begin{aligned}
&\|T(t, s) P(s)\| \leq K t^{n} e_{\alpha}(t, s), t \geq s \geq \eta>0, \\
&\|T(t, s) Q(s)\| \leq K s^{n} e_{\beta}(t, s), \quad s \geq t,
\end{aligned}
$$

and

$$
\int_{t_{0}}^{\infty} \sigma^{n}(s) h(s)<\infty
$$

If $x(t)$ is a solution of (2) with Lyapunov exponent $\alpha$, then $\frac{\|x(t)\|}{t^{n} e_{\alpha}\left(t, t_{0}\right)}$ is bounded for $t \geq \eta$. 
Proof If $x(t)$ is a solution for (2) with Lyapunov number $\alpha$, then, for $\varepsilon>0$ with $\alpha<\alpha+\varepsilon<\beta$ and $0<\delta<\varepsilon$, there exists $L>0$ such that

$$
\|x(t)\| \leq L e_{\alpha+\delta}(t) \quad \text { and } \quad\|T(t, s) Q(s)\| \leq K s^{n} e_{\alpha+\delta}(t, s), \quad s \geq t .
$$

On the other hand,

$$
\begin{aligned}
x(t)= & T(t, \eta) \omega+\int_{\eta}^{t} T(t, \sigma(s)) P(\sigma(s)) f(s, x(s)) \Delta s \\
& -\int_{t}^{\infty} T(t, \sigma(s)) Q(\sigma(s)) f(s, x(s)) \Delta s \\
= & T(t, \eta) P(\eta) \omega+T(t, \eta) Q(\eta) \omega+\int_{\eta}^{t} T(t, \sigma(s)) P(\sigma(s)) f(s, x(s)) \Delta s \\
& -\int_{t}^{\infty} T(t, \sigma(s)) Q(\sigma(s)) f(s, x(s)) \Delta s,
\end{aligned}
$$

where $\omega=x(\eta)+\int_{\eta}^{\infty} T(\eta, \sigma(s)) Q(\sigma(s)) f(s, x(s)) \Delta s$.

Proceeding as in the previous lemma, we obtain

$$
\begin{aligned}
& \left\|\int_{t}^{\infty} T(t, \sigma(s)) Q(\sigma(s)) f(s, x(s)) \Delta s\right\| \\
& \quad \leq \int_{t}^{\infty}\|T(t, \sigma(s)) Q(\sigma(s))\|\|f(s, x(s))\| \Delta s \\
& \quad \leq \int_{t}^{\infty} K \sigma^{n}(s) e_{\alpha+\delta}(t, \sigma(s)) h(s)\|x(s)\| \Delta s \\
& \quad \leq K e_{\alpha+\delta}\left(t, t_{0}\right) \int_{t}^{\infty} e_{\ominus(\alpha+\delta)}\left(s, t_{0}\right) \sigma^{n}(s) h(s)\|x(s)\| \Delta s \\
& \quad \leq K L e_{\alpha+\delta}\left(t, t_{0}\right) \int_{t}^{\infty} \sigma^{n}(s) h(s) \Delta s<\infty .
\end{aligned}
$$

So, the above integrals are convergent. Analogously, we get the following estimate:

$$
\begin{aligned}
\left\|\int_{\eta}^{t} T(t, \sigma(s)) P(\sigma(s)) f(s, x(s)) \Delta s\right\| & \leq \int_{\eta}^{t}\|T(t, \sigma(s)) P(\sigma(s))\|\|f(s, x(s))\| \Delta s \\
& \leq K t^{n} e_{\alpha}\left(t, t_{0}\right) \int_{\eta}^{t} e_{\ominus \alpha}\left(s, t_{0}\right) h(s)\|x(s)\| \Delta s .
\end{aligned}
$$

Therefore,

$$
\begin{aligned}
T(t, \eta) \omega= & x(t)-\int_{\eta}^{t} T(t, \sigma(s)) P(\sigma(s)) f(s, x(s)) \Delta s \\
& +\int_{t}^{\infty} T(t, \sigma(s)) Q(\sigma(s)) f(s, x(s)) \Delta s
\end{aligned}
$$


and

$$
\begin{gathered}
\|T(t, \eta) \omega\| \leq\|x(t)\|+\int_{\eta}^{t}\|T(t, \sigma(s)) P(\sigma(s)) f(s, x(s))\| \Delta s \\
+\int_{t}^{\infty}\|T(t, \sigma(s)) Q(\sigma(s)) f(s, x(s))\| \Delta s
\end{gathered}
$$

which implies that

$$
\begin{aligned}
\frac{\|T(t, \eta) \omega\|}{t^{n} e_{\alpha+\delta}\left(t, t_{0}\right)} \leq & \frac{\|x(t)\|}{t^{n} e_{\alpha+\delta}\left(t, t_{0}\right)}+\frac{1}{t^{n} e_{\alpha+\delta}\left(t, t_{0}\right)} \int_{\eta}^{t}\|T(t, \sigma(s)) Q(\sigma(s)) f(s, x(s))\| \Delta s \\
& +\frac{1}{t^{n} e_{\alpha+\delta}\left(t, t_{0}\right)} K e_{\alpha+\delta}\left(t, t_{0}\right) \int_{t}^{\infty} e_{\ominus(\alpha+\delta)}\left(s, t_{0}\right) \sigma^{n}(s) h(s)\|x(s)\| \Delta s \\
\leq & \frac{\|x(t)\|}{t^{n} e_{\alpha+\delta}\left(t, t_{0}\right)}+K e_{\alpha \ominus(\alpha+\delta)}\left(t, t_{0}\right) \int_{\eta}^{t} e_{\ominus \alpha}\left(s, t_{0}\right) h(s)\|x(s)\| \Delta s \\
& +\frac{K}{t^{n}} \int_{t}^{\infty} e_{\ominus(\alpha+\delta)}\left(s, t_{0}\right) \sigma^{n}(s) h(s)\|x(s)\| \Delta s \longrightarrow 0, \quad \text { as } t \rightarrow \infty .
\end{aligned}
$$

Analogously to the previous lemma, we can prove that $Q(\eta) \omega=0$. So, $P(\eta) \omega=\omega$ and

$$
\begin{aligned}
x(t)= & T(t, \eta) P(\eta) \omega+\int_{\eta}^{t} T(t, \sigma(s)) P(\sigma(s)) f(s, x(s)) \Delta s \\
& -\int_{t}^{\infty} T(t, \sigma(s)) Q(\sigma(s)) f(s, x(s)) \Delta s .
\end{aligned}
$$

Therefore,

$$
\begin{aligned}
\frac{\|x(t)\|}{t^{n} e_{\alpha}\left(t, t_{0}\right)} \leq & \frac{\|T(t, \eta) P(\eta) \omega\|}{t^{n} e_{\alpha}\left(t, t_{0}\right)}+\frac{1}{t^{n} e_{\alpha}\left(t, t_{0}\right)} \int_{\eta}^{t}\|T(t, \sigma(s)) P(\sigma(s))\|\|f(s, x(s))\| \Delta s \\
& +\frac{1}{t^{n} e_{\alpha}\left(t, t_{0}\right)} \int_{t}^{\infty}\|T(t, \sigma(s)) Q(\sigma(s))\|\|f(s, x(s))\| \Delta s \\
\leq & \frac{K t^{n} e_{\alpha}(t, \eta)}{t^{n} e_{\alpha}\left(t, t_{0}\right)}\|\omega\|+\frac{1}{t^{n} e_{\alpha}\left(t, t_{0}\right)} \int_{\eta}^{t} K t^{n} e_{\alpha}(t, \sigma(s)) h(s)\|x(s)\| \Delta s \\
& +\frac{1}{t^{n} e_{\alpha}\left(t, t_{0}\right)} \int_{t}^{\infty} K e_{\alpha+\delta}(t, \sigma(s)) \sigma^{n}(s) h(s)\|x(s)\| \Delta s \\
\leq & K e_{\ominus \alpha}\left(\eta, t_{0}\right)\|\omega\|+K \int_{\eta}^{t} s^{n} h(s) \frac{\|x(s)\|}{s^{n} e_{\alpha}\left(s, t_{0}\right)} \Delta s \\
& +\frac{K}{t^{n} e_{\alpha \ominus(\alpha+\delta)}\left(t, t_{0}\right)} \int_{t}^{\infty} s^{n} e_{\alpha \ominus(\alpha+\delta)}\left(s, t_{0}\right) \sigma^{n}(s) h(s) \frac{\|x(s)\|}{s^{n} e_{\alpha \ominus(\alpha+\delta)}\left(s, t_{0}\right)} \Delta s .
\end{aligned}
$$

Now, if we put

$$
u(t)=\frac{\|x(t)\|}{t^{n} e_{\alpha}\left(t, t_{0}\right)}, \quad \gamma(t)=t^{n} e_{\alpha \ominus(\alpha+\delta)}\left(t, t_{0}\right), \quad f(t)=t^{n} h(t), \quad g(t)=\sigma^{n}(t) h(t),
$$

then, applying Lemma 4.2, we obtain that

$$
u(t) \leq \frac{K e_{\ominus \alpha}\left(\eta, t_{0}\right)\|\omega\|}{1-\beta} \exp \left\{\frac{1}{1-\beta} \int_{t}^{\infty} \ominus(-g(s)) \Delta s\right\}
$$


where

$$
\beta=\int_{\eta}^{\infty} f(s) \Delta s+\int_{\eta}^{\infty} g(s) \Delta s<1
$$

Therefore, $\frac{\|x(t)\|}{t^{n} e_{\alpha}\left(t, t_{0}\right)}$ is bounded.

The following theorem gives answer to the converse problem.

Theorem 4.1 Suppose that $T(t, s)$ is the evolution operator generated by $A(t)$ and assume that the following statements hold:

(a) For $\alpha \in \mathbb{R}$ and $N \in \mathbb{N}, N>1$ fixed, system (1) has solution $y(t)$ with Lyapunov exponent $\alpha$ and there exists an integer $l$ with $0 \leq l \leq N-1$ such that $y(t) \cong t^{l} e_{\alpha}\left(t, t_{0}\right)$.

(b) For each $l, 0 \leq l \leq N-1$, system (1) has a generalized polynomial exponential trichotomy on time scales as in Definition 3.1.

(c) $\int_{t_{0}}^{\infty} \sigma^{N}(s) h(s) \Delta s<\infty$.

Then, if $x(t)$ is a solution of (2) with Lyapunov exponent $\alpha$ then there exists a solution $y(t)$ of (1) such that

$$
\lim _{t \rightarrow \infty} \frac{\|x(t)-y(t)\|}{\|x(t)\|}=0
$$

Moreover, there exists $\bar{l}, 0 \leq \bar{l} \leq N-1$ such that $x(t) \cong t^{\bar{l}} e_{\alpha}\left(t, t_{0}\right)$.

Proof From Definition 3.1 we have that $P(t), S(t), Q(t), U(t)$ are complementary projections and $(P(t)+S(t))+(Q(t)+U(t))=I$, so, if $\bar{P}(t)=P(t)+S(t)$ and $\bar{Q}(t)=Q(t)+U(t)$, then

$$
\begin{aligned}
\|T(t, s) \bar{P}(s)\| & \leq M t^{l-1} s^{N-l} e_{\alpha}(t, s)+M e_{\alpha \ominus \varepsilon}(t, s), \\
& \leq 2 M t^{N-1} e_{\alpha}(t, s), \quad t \geq s . \\
\|T(t, s) \bar{Q}(s)\| & \leq M t^{l} s^{N-l-1} e_{\alpha}(t, s)+M e_{\alpha \oplus \varepsilon}(t, s), \\
& \leq 2 M s^{N-1} e_{\alpha}(t, s), \quad s \geq t,
\end{aligned}
$$

which implies that the conditions from Lemma 4.3 are satisfied and therefore $\frac{\|x(t)\|}{t^{N-1} e_{\alpha}\left(t, t_{0}\right)}$ is bounded.

Let us define

$$
l=\min \left\{m \in\{0, \ldots, N-1\}: \frac{\|x(t)\|}{t^{m} e_{\alpha}\left(t, t_{0}\right)} \text { is bounded for } t \geq \eta\right\} .
$$

As in Lemma 4.3, if we put

$$
y(t)=T(t, \eta)\left[x(\eta)+\int_{\eta}^{\infty} T(\eta, \sigma(s))(Q(\sigma(s))+U(\sigma(s)) f(s, x(s)) \Delta s],\right.
$$


for $t \geq \eta$, we obtain that $y(t)$ is a solution of $(1)$ and

$$
\begin{aligned}
x(t)= & y(t)+\int_{\eta}^{t} T(t, \sigma(s))[P(\sigma(s))+S(\sigma(s))] f(s, x(s)) \Delta s \\
& -\int_{t}^{\infty} T(t, \sigma(s))[Q(\sigma(s))+U(\sigma(s))] f(s, x(s)) \Delta s .
\end{aligned}
$$

Therefore,

$$
\begin{aligned}
\|x(t)-y(t)\| \leq & \int_{\eta}^{t}[\|T(t, \sigma(s)) P(\sigma(s))\|+\|T(t, \sigma(s)) S(\sigma(s))\|]\|f(s, x(s))\| \Delta s \\
& +\int_{t}^{\infty}[\|T(t, \sigma(s)) Q(\sigma(s))\|+\|T(t, \sigma(s)) U(\sigma(s))\|]\|f(s, x(s))\| \Delta s \\
\leq & \int_{\eta}^{t}\left[M t^{l-1} \sigma^{N-l}(s) e_{\alpha}(t, \sigma(s))+M e_{\alpha \ominus \varepsilon}(t, \sigma(s))\right] h(s)\|x(s)\| \Delta s \\
& +\int_{t}^{\infty}\left[M t^{l} \sigma^{N-l-1}(s) e_{\alpha}(t, \sigma(s))+M e_{\alpha \oplus \varepsilon}(t, \sigma(s))\right] h(s)\|x(s)\| \Delta s \\
\leq & t^{l-1} e_{\alpha}\left(t, t_{0}\right) \int_{\eta}^{t} M\left[\sigma^{N}(s)+(1+\sigma(s) \varepsilon) \frac{\sigma^{l}(s)}{t^{l-1}} e_{\ominus \varepsilon}(t, s)\right] h(s) \frac{\|x(s)\|}{s^{l} e_{\alpha}\left(s, t_{0}\right)} \Delta s \\
& +t^{l} e_{\alpha}\left(t, t_{0}\right) \int_{t}^{\infty} M\left[\sigma^{N-1}(s)+\frac{\sigma^{l}(s)}{t^{l}} e_{\varepsilon}(t, s)\right] h(s) \frac{\|x(s)\|}{s^{l} e_{\alpha}\left(s, t_{0}\right)} \Delta s \\
\leq & t^{l-1} e_{\alpha}\left(t, t_{0}\right)\|x\|_{\alpha, l} \int_{\eta}^{t} M\left[\sigma^{N}(s)+(1+\sigma(s) \varepsilon) \frac{\sigma^{l}(s)}{\left.t^{l-1}\right] h(s) \Delta s}\right. \\
& +t^{l} e_{\alpha}\left(t, t_{0}\right)\|x\|_{\alpha, l} \int_{t}^{\infty} M\left[\sigma^{N-1}(s)+\frac{\sigma^{l}(s)}{t^{l}}\right] h(s) \Delta s,
\end{aligned}
$$

where $\|x\|_{\alpha, l}=\sup _{t>\eta} \frac{\|x(t)\|}{t^{l} e_{\alpha}\left(t, t_{0}\right)}$. So,

$$
\begin{aligned}
\frac{\|x(t)-y(t)\|}{t^{l} e_{\alpha}\left(t, t_{0}\right)} \leq & M\|x\|_{\alpha, l}\left(\frac{1}{t} \int_{\eta}^{t}\left[\sigma^{N}(s)(1+\sigma(s) \varepsilon) \frac{\sigma^{l}(s)}{\eta^{l}}\right] h(s) \Delta s\right. \\
& \left.+\int_{t}^{\infty}\left[\sigma^{N-1}(s)+\frac{\sigma^{l}(s)}{\eta^{l}}\right] h(s) \Delta s\right) .
\end{aligned}
$$

From assumption (c) we obtain that $\lim _{t \rightarrow \infty} \frac{\|x(t)-y(t)\|}{t^{l} e_{\alpha}\left(t, t_{0}\right)}=0$. This implies that $\frac{\|y(t)\|}{t^{l} e_{\alpha}(t)}$ is bounded for $t \geq \eta$.

If we suppose first that $l=0$, then in this case $\|x(t)\| / e_{\alpha}\left(t, t_{0}\right)$ is bounded for $t \geq \eta$ and $P(s) \equiv 0$.

From (26) it follows, for $t \geq \eta$, that

$$
\begin{aligned}
x(t)= & T(t, \eta) \omega+\int_{\eta}^{t} T(t, \sigma(s)) S(\sigma(s)) f(s, x(s)) \Delta s \\
& -\int_{t}^{\infty}[Q(\sigma(s))+U(\sigma(s))] f(s, x(s)) \Delta s
\end{aligned}
$$

where $\omega=x(\eta)+\int_{\eta}^{t} T(\eta, \sigma(s))[Q(\sigma(s))+U(\sigma(s))] f(s, x(s)) \Delta x$. 
We claim that $[Q(\eta)+U(\eta)] \omega \neq 0$. In fact, if this it is not true, then we have $S(\eta) \omega=\omega$ and so

$$
\|T(t, \eta) \omega\|=\|T(t, \eta) S(\eta) \omega\| \leq M e_{\alpha \ominus \varepsilon}(t, \eta)\|\omega\|, \quad t \geq \eta .
$$

Therefore

$$
\begin{aligned}
\|x(t)\| \leq & \|T(t, \eta) \omega\|+\int_{\eta}^{t}\|T(t, \sigma(s)) S(\sigma(s))\| h(s)\|x(s)\| \Delta s \\
& +\int_{\eta}^{\infty}\|T(t, \sigma(s))[Q(\sigma(s))+U(\sigma(s))]\| h(s)\|x(s)\| \Delta s \\
\leq & M e_{\alpha \ominus \varepsilon}(t, \eta)\|\omega\|+\int_{\eta}^{t} M(1+\sigma(s) \varepsilon) e_{\alpha \ominus \varepsilon}(t, s) h(s)\|x(s)\| \Delta s \\
& +\int_{t}^{\infty} M \sigma^{N-1}(s) e_{\alpha}(t, s) h(s)\|x(s)\| \Delta s+\int_{t}^{\infty} M e_{\alpha \oplus \varepsilon}(t, s) h(s)\|x(s)\| \Delta s .
\end{aligned}
$$

Now,

$$
\begin{aligned}
\frac{\|x(t)\|}{e_{\alpha \ominus \varepsilon}\left(t, t_{0}\right)} \leq & M e_{\alpha \ominus \varepsilon}\left(t_{0}, \eta\right)\|\omega\|+\int_{\eta}^{t} M(1+\sigma(s) \varepsilon) e_{\ominus(\alpha \ominus \varepsilon)}\left(s, t_{0}\right) h(s)\|x(s)\| \Delta s \\
& +\frac{1}{e_{\ominus \varepsilon}\left(t, t_{0}\right)} \int_{t}^{\infty} e_{\ominus \varepsilon}\left(s, t_{0}\right)\left(\sigma^{N-1}(s)+1\right) M e_{\ominus(\alpha \ominus \varepsilon)}\left(s, t_{0}\right) h(s)\|x(s)\| \Delta s .
\end{aligned}
$$

By using Lemma 4.2, we have that $\|x(s)\| / e_{\alpha \ominus \varepsilon}\left(t, t_{0}\right)$ is bounded for $t \geq \eta$, therefore there exists $L>0$ such that $\|x(t)\| \leq L e_{\alpha \ominus \varepsilon}\left(t, t_{0}\right) \leq L e_{\alpha-\varepsilon}\left(t, t_{0}\right)$, but this contradicts the fact that $x(t)$ has Lyapunov exponent $\alpha$.

Since $\|y(t)\| / e_{\alpha}\left(t, t_{0}\right)$ is bounded for $t \geq \eta$, we obtain

$$
0<\liminf _{t \rightarrow \infty} \frac{\|y(t)\|}{e_{\alpha}\left(t, t_{0}\right)} \leq \limsup _{t \rightarrow \infty} \frac{\|y(t)\|}{e_{\alpha}\left(t, t_{0}\right)}<\infty
$$

and

$$
0<\liminf _{t \rightarrow \infty} \frac{\|x(t)\|}{e_{\alpha}\left(t, t_{0}\right)} \leq \limsup _{t \rightarrow \infty} \frac{\|x(t)\|}{e_{\alpha}\left(t, t_{0}\right)}<\infty .
$$

Hence

$$
\lim _{t \rightarrow \infty} \frac{\|x(t)-y(t)\|}{\|x(t)\|}=\lim _{t \rightarrow \infty} \frac{\|x(t)-y(t)\| / e_{\alpha}\left(t, t_{0}\right)}{\|x(t)\| / e_{\alpha}\left(t, t_{0}\right)}=\lim _{t \rightarrow \infty} \frac{\|x(t)-y(t)\|}{e_{\alpha}\left(t, t_{0}\right)}=0 .
$$

Now, if $l \geq 1$, from (26) we obtain

$$
\begin{aligned}
\|x(t)\| \leq & \|y(t)\|+\int_{\eta}^{t}\|T(t, \sigma(s))[P(\sigma(s))+S(\sigma(s))]\|\|f(x, x(s))\| \Delta s \\
& +\int_{t}^{\infty}\|T(t, \sigma(s))[Q(\sigma(s))+U(\sigma(s))]\|\|f(s, x(s))\| \Delta s \\
\leq & \|y(t)\|+\int_{\eta}^{t} M e_{\alpha} e(t, \sigma(s))\left[t^{l-1} \sigma^{N-l}(s)+e_{\ominus \varepsilon}(t, \sigma(s))\right] h(s)\|x(s)\| \Delta s
\end{aligned}
$$




$$
\begin{aligned}
& +\int_{t}^{\infty} M e_{\alpha}(t, \sigma(s))\left[t^{l} \sigma^{N-l-1}(s)+e_{\varepsilon}(t, \sigma(s))\right] h(s)\|x(s)\| \Delta s \\
\leq & \|y(t)\|+\int_{\eta}^{t} M e_{\alpha}(t, s)\left[t^{l-1} \sigma^{N-l}(s)+(1+\sigma(s) \varepsilon) e_{\ominus \varepsilon}(t, s)\right] h(s)\|x(s)\| \Delta s \\
& +\int_{t}^{\infty} e_{\alpha}(t, s)\left[t^{l} \sigma^{N-l-1}(s)+e_{\varepsilon}(t, s)\right] h(s)\|x(s)\| \Delta s .
\end{aligned}
$$

We claim that $\frac{\|y(t)\|}{t^{l-1} e_{\alpha}\left(t, t_{0}\right)}$ is not bounded. Let us suppose that $\frac{\|y(t)\|}{t^{l-1} e_{\alpha}\left(t, t_{0}\right)} \leq L$ for some $L>0$. Then, we have that

$$
\begin{aligned}
& \frac{\|x(t)\|}{t^{l-1} e_{\alpha}\left(t, t_{0}\right)} \\
& \leq \frac{\|y(t)\|}{t^{l-1} e_{\alpha}\left(t, t_{0}\right)} \\
& \quad+\frac{1}{t^{l-1} e_{\alpha}\left(t, t_{0}\right)} \int_{\eta}^{t} M e_{\alpha}(t, s)\left[t^{l-1} \sigma^{N-l}(s)+(1+\sigma(s) \varepsilon) e_{\ominus \varepsilon}(t, s)\right] h(s)\|x(s)\| \Delta s \\
& \quad+\frac{1}{t^{l-1} e_{\alpha}\left(t, t_{0}\right)} \int_{t}^{\infty} M e_{\alpha}(t, s)\left[t^{l} \sigma^{N-l-1}(s)+e_{\varepsilon}(t, s)\right] h(s)\|x(s)\| \Delta s \\
& \leq L \\
& +\int_{\eta}^{t} M\left[\sigma^{N-l}(s) s^{l-1}+(1+\sigma(s) \varepsilon)\right] h(s) \frac{\|x(s)\|}{s^{l-1} e_{\alpha}\left(s, t_{0}\right)} \Delta s \\
& \quad+t \int_{t}^{\infty} \frac{M}{s}\left[\sigma^{N-l-1}(s) s^{l}+1\right] h(s) \frac{\|x(s)\|}{s^{l-1} e_{\alpha}\left(s, t_{0}\right)} \Delta s \\
& \leq L+\int_{\eta}^{t} M(2+\varepsilon) \sigma^{N-1}(s) h(s) \frac{\|x(s)\|}{s^{l-1} e_{\alpha}\left(s, t_{0}\right)} \Delta s \\
& \quad+t \int_{t}^{\infty} 2 \frac{M}{s} \sigma^{N-1}(s) h(s) \frac{\|x(s)\|}{s^{l-1} e_{\alpha}\left(s, t_{0}\right)} \Delta s .
\end{aligned}
$$

From Lemma 4.2 it follows that $\frac{\|x(t)\|}{t^{l-1} e_{\alpha}(t)}$ is bounded, contradicting the definition of $l$. So

$$
0<\liminf _{t \rightarrow \infty} \frac{\|y(t)\|}{t^{l} e_{\alpha}\left(t, t_{0}\right)} \leq \limsup _{t \rightarrow \infty} \frac{\|y(t)\|}{t^{l} e_{\alpha}\left(t, t_{0}\right)}<\infty
$$

and

$$
0<\liminf _{t \rightarrow \infty} \frac{\|x(t)\|}{t^{l} e_{\alpha}\left(t, t_{0}\right)} \leq \limsup _{t \rightarrow \infty} \frac{\|x(t)\|}{t^{l} e_{\alpha}\left(t, t_{0}\right)}<\infty .
$$

Thus

$$
\lim _{t \rightarrow \infty} \frac{\|x(t)-y(t)\|}{\|x(t)\|}=\lim _{t \rightarrow \infty} \frac{\|x(t)-y(t)\| / t^{l} e_{\alpha}\left(t, t_{0}\right)}{\|x(t)\| / t^{l} e_{\alpha}\left(t, t_{0}\right)}=\lim _{t \rightarrow \infty} \frac{\|x(t)-y(t)\|}{t^{l} e_{\alpha}\left(t, t_{0}\right)}=0 .
$$

This concludes the proof.

\section{Examples}

Example 5.1 Let us consider the dynamic equation

$$
x^{\Delta}(t)=A x(t)+f(t, x(t)), \quad t \in[0, \infty)_{\mathbb{T}},
$$


where $A=\left(\begin{array}{ll}1 & 0 \\ 1 & 1\end{array}\right)$ and $f: \mathbb{T} \times \mathbb{R}^{2} \longrightarrow \mathbb{R}^{2}$ satisfying condition (3).

We compare system (27) with the unperturbed system

$$
y^{\Delta}(t)=A y(t), \quad t \in[0, \infty)_{\mathbb{T}},
$$

The eigenvalues of the matrix $A$ are $\lambda_{1}=\lambda_{2}=1$, therefore $1+\mu(t) \neq 0$, so $A$ is regressive for any time scale $\mathbb{T}$. Hence, is easy to see that the evolution operator associated to (28) is given by

$$
T(t, s)=e_{A}(t, s)=e_{1}(t, s)\left(\begin{array}{cc}
1 & 0 \\
\int_{s}^{t} \frac{\Delta \tau}{1+\mu(t)} & 1
\end{array}\right) .
$$

For example,

(1) if $\mathbb{T}=\mathbb{R}$, then $T(t, s)=e^{t-s}\left(\begin{array}{cc}1 & 0 \\ t-s & 1\end{array}\right)$,

(2) if $\mathbb{T}=\mathbb{Z}$, then $T(t, s)=2^{t-s}\left(\begin{array}{cc}1 & 0 \\ \frac{1}{2}(t-s) & 1\end{array}\right)$,

(3) if $\mathbb{T}=h \mathbb{Z}$, then $T(t, s)=(1+h)^{\frac{t-s}{h}}\left(\begin{array}{rr}\frac{1}{1+h}(t-s) & 0\end{array}\right)$,

Now, let us define the projections

$$
P(s)=e_{A}(s, 0)\left(\begin{array}{ll}
0 & 0 \\
0 & 1
\end{array}\right) e_{A}^{-1}(s, 0) \text { and } Q(s)=e_{A}(s, 0)\left(\begin{array}{ll}
1 & 0 \\
0 & 0
\end{array}\right) e_{A}^{-1}(s, 0),
$$

therefore,

$$
\begin{array}{ll}
\|T(t, s) P(s)\| \leq M s e_{1}(t, s), \quad t \geq s \geq \beta, \\
\|T(t, s) Q(s)\| \leq M t e_{1}(t, s), \quad s \geq t \geq \beta,
\end{array}
$$

with $l=1, N=2, \alpha=1$, and $S(s)=U(s)=0$; moreover, $\|y(t)\| \cong t e_{1}(t, 0)$. If $\int_{0}^{\infty} \sigma(s) h(s) \Delta s<$ $\infty$, then, by Theorem 3.1, system (27) has a solution $x(t)$ such that

$$
\lim _{t \rightarrow \infty} \frac{\|x(t)-y(t)\|}{\|y(t)\|}=0
$$

Example 5.2 Let $\mathbb{T}=\left\{2^{n}: n \in \mathbb{N}^{0}\right\}$, where $\mathbb{N}^{0}$ is the set of nonnegative integers.

Consider the following system:

$$
x^{\Delta}(t)=A x(t)+f(t, x(t)), \quad t \in[1, \infty)_{\mathbb{T}},
$$

where $A=\left(\begin{array}{cc}-3 & -2 \\ 3 & 4\end{array}\right)$ and $f(t, x)=\left(\frac{0}{\frac{\sin (x(\sigma(t)))}{\sigma(t) t}}\right)$. The eigenvalues of $A$ are $\lambda_{1}=-2$ and $\lambda=3$. Since $1-2 \mu(t)=1-2 t \neq 0$ for all $t \in \mathbb{T}$, we get that $A$ is regressive.

The evolution operator associated to the linear system

$$
y^{\Delta}(t)=A y(t), \quad t \in[1, \infty)_{\mathbb{T}},
$$

is given by

$$
T(t, s)=e_{A}(t, s)=\left(\begin{array}{cc}
2 e_{-2}(t, s) & e_{3}(t, s) \\
-e_{-2}(t, s) & -3 e_{3}(t, s)
\end{array}\right)=\left(\begin{array}{cc}
2 \prod_{\tau \in[s, t)}(1-2 \tau) & \prod_{\tau \in[s, t)}(1+3 \tau) \\
-\prod_{\tau \in[s, t)}(1-2 \tau) & -3 \prod_{\tau \in[s, t)}(1+3 \tau)
\end{array}\right) .
$$


If we define the projections

$$
P(s)=e_{A}(s, 1)\left(\begin{array}{ll}
0 & 0 \\
0 & 1
\end{array}\right) e_{A}^{-1}(s, 1) \text { and } Q(s)=e_{A}(s, 1)\left(\begin{array}{ll}
1 & 0 \\
0 & 0
\end{array}\right) e_{A}^{-1}(s, 1),
$$

then

$$
\begin{aligned}
&\|T(t, s) P(s)\| \leq \frac{8}{5} e_{3}(t, s), \quad t \geq s \geq \beta, \\
&\|T(t, s) Q(s)\| \leq \frac{8}{5} e_{3}(t, s), \quad s \geq t \geq \beta .
\end{aligned}
$$

We have that $l=0, N=1, \alpha=3, S(s)=0$, and $U(s)=0$. Since $y(t) \cong e_{3}(t, 1)$ and $\int_{1}^{\infty} h(s) \Delta s=\int_{1}^{\infty} \frac{\Delta s}{\sigma(s) s}<\infty$, by Theorem 3.1, system (29) has a solution $x(t)$ such that

$$
\lim _{t \rightarrow \infty} \frac{\|x(t)-y(t)\|}{\|y(t)\|}=0 .
$$

\section{Concluding remarks}

In this paper we studied the asymptotic relative equivalence between the solutions of two dynamic equations on time scales, one linear and the other formed by its nonlinear perturbation, unifying and extending the results presented in the references. To accomplish this goal, we introduced the concept of a polynomial exponential trichotomy on time scales which is a generalization of the concept of polynomial exponential dichotomy presented by Leiva and Rodrigues in [16]. It is important to mention that this concept is more general than the simple concept of polynomial dichotomy, as it involves several projections that appear in a natural way when one breaks the Jordan decomposition block in order to study a relative behavior of solutions of differential equations. In a forthcoming paper we are going to show the existence and roughness of this type of trichotomy.

\section{Acknowledgements}

The authors would like to thank the anonymous reviewer for his/her valuable suggestions, comments, and criticism for improving the quality of this paper.

\section{Funding}

No funding.

\section{Availability of data and materials}

Data sharing not applicable to this article as no datasets were generated or analyzed during the current study.

\section{Declarations}

Competing interests

The authors declare that they have no competing interests.

\section{Authors' contributions}

All authors contributed equally to this work. All authors read and approved the final manuscript.

Author details

${ }^{1}$ Facultad de Ciencias, Departamento de Matemáticas, Universidad de Los Andes, Mérida, Venezuela. ${ }^{2}$ School of Mathematical and Computational Siences, University YachayTech, San Miguel de Urcuqui, Imbabura, Ecuador.

${ }^{3}$ Department Mathematical Sciences, United Arab Emirates University, Al Ain, UAE.

\section{Publisher's Note}

Springer Nature remains neutral with regard to jurisdictional claims in published maps and institutional affiliations. 


\section{References}

1. Agarwal, R., O'Regan, D., Saker, S.: Dynamic Inequalities on Time Scales. Springer, Berlin (2014)

2. Atici, F.M., Biles, D.C., Lebedinsky, A.: An application of time scales to economics. Math. Comput. Model. 43, 718-726 (2006)

3. Bohner, M., Fan, M., Zhang, J.: Existence of periodic solutions in predator-prey and competition dynamic systems. Nonlinear Anal., Real World Appl. 7, 1193-1204 (2006)

4. Bohner, M., Peterson, A.: Dynamics Equations on Time Scales. An Introduction with Applications. Birkhäuser, Boston (2001)

5. Bohner, M., Peterson, A.: Advances in Dynamic Equations on Time Scales. Birkhäuser, Boston (2003)

6. Brauer, F., Wong, J.S.: On the asymptotic relationships between solutions of two systems of ordinary differential differential equations. J. Differ. Equ. 6, 527-543 (1969)

7. Duque, C., Uzcátegui, J., Leiva, H.: Controllability of semilinear dynamic systems on time scales. J. Abstr. Differ. Equ. Appl. 8(2), 70-80 (2017)

8. Guseinov, G.S.: Integration on time scales. J. Math. Anal. Appl. 285, 107-127 (2003)

9. Hamza, A., Oraby, K.: Stability of abstract dynamic equations on time scales by Lyapunov's second method. Turk. J. Math. 42, 841-861 (2018)

10. Hamza, A.E., Oraby, K.M.: On the exponential operator functions on time scales. Adv. Dyn. Syst. Appl. 7(1), 57-80 (2012)

11. Hamza, A.E., Oraby, K.M.: Characterization of stability of abstract dynamic equations on time scales. Commum. Korean Math. Soc. 34(1), 185-202 (2019)

12. Hilger, S.: Ein Maßkettenkalkül mit Anwendung auf Zentrumsmanningfaltigkeiten. PhD thesis, Universität Würzburg (1988)

13. Hilger, S.: Analysis on measure chains - a unified approach to continuous and discrete calculus. Results Math. 18, 18-56 (1990)

14. Hilger, S.: Differential and difference calculus- unified! Nonlinear Anal. 30(5), 2683-2694 (1997)

15. Leiva, H., Medina, E., Merentes, N.: Relative asymptotic equivalence between difference equations. J. Differ. Equ. Appl. 21(5), 418-436 (2015)

16. Leiva, H., Rodrigues, H.M.: Relative asymptotic equivalence of evolution equations. Nonlinear Anal. 47, 4579-4590 (2001)

17. Li, Y., Wang, C.: Almost periodic functions on time scales and applications. Discrete Dyn. Nat. Soc. 2011, Article ID $727068(2011)$

18. Li, Y., Wang, C.: Uniformly almost periodic functions and almost periodic solutions to dynamic equations on time scales. Abstr. Appl. Anal. 2011, Article ID 341520 (2011)

19. Pachpatte, B.G.: Inequalities for Differential and Integral Equations. Mathematics in Science and Engineering, vol. 197. Academic Press, San Diego (1998)

20. Pötzsche, C.: Exponential dichotomies for dynamic equations on measure chains. Nonlinear Anal., Theory Methods Appl. 47, 873-884 (2001)

21. Pötzsche, C.: Exponential dichotomies of linear dynamic equations on measure chains under slowly varying coefficients. J. Math. Anal. Appl. 289, 317-335 (2004)

22. Pötzsche, C., Siegmund, S., Wirth, F.: A spectral characterization of exponential stability for linear time-invariant systems on time scales. Discrete Contin. Dyn. Syst. 9(5), 1223-1241 (2003)

23. Rodrigues, H.M.: Relative asymptotic equivalence with weight between two systems of ordinary differential equations. In: Dynamical Systems and International Symposium 2, pp. 249-254. Academic Press, New York (1976)

24. Rodrigues, H.M.: On growth and decay of solutions of perturbed retarded linear equations. Tohoku Math. J. 32, 32593-32605 (1980)

25. Yang, L., Zhang, J., Chang, X., Liu, Z.: Exponential dichotomy on time scales and admissibility of the pair $\left(C_{r d}\left(\mathbb{T}^{+}, X\right), L^{p}\left(\mathbb{T}^{+}, X\right)\right)$. Adv. Differ. Equ. 2015, 69 (2015). https://doi.org/10.1186/s13662-015-0409-7

26. Zhang, J., Fan, M., Zhu, H.: Existence and roughness of exponential dichotomies of linear dynamic equations on time scales. Comput. Math. Appl. 59, 2658-2675 (2010)

27. Zhang, J., Song, Y., Zhao, Z.: General exponential dichotomies on time scales and parameter dependence of roughness. Adv. Differ. Equ. 2013, 339 (2013)

\section{Submit your manuscript to a SpringerOpen ${ }^{\circ}$ journal and benefit from:}

- Convenient online submission

- Rigorous peer review

- Open access: articles freely available online

- High visibility within the field

- Retaining the copyright to your article

Submit your next manuscript at $\gg$ springeropen.com 